\title{
Incommensurate instability and lattice dynamics of potassium selenate within a semiempirical rigid-ion model
}

\author{
I. Etxebarria and J. M. Perez-Mato \\ Departamento de Física de la Materia Condensada, Facultad de Ciencias, Universidad del Pais Vasco, \\ Apartado Postal 644, 48080 Bilbao, Spain
}

A. Criado

Departamento de Física de la Materia Condensada, Facultad de Ciencias Físicas, Universidad de Sevilla, Apartado Postal 1065, 41080 Sevilla, Spain

(Received 23 May 1990)

\begin{abstract}
The lattice dynamics of potassium selenate is analyzed using a rigid-ion model with the selenate groups reduced to rigid bodies. The interatomic forces have been adjusted only using static structural data. The number of adjustable parameters varies from two to five. Such a simple model is already sufficient to reproduce semiquantitatively the phonon dynamics of the real system. In particular, the model exhibits the lattice instability leading to the existence of an incommensurate phase. The characteristics of the resulting soft mode agree with those observed experimentally. The calculated eigenvector, in excellent agreement with the experimental one, is rather unsensitive to the details of the interactions. This explains the strong similarities of the incommensurate modulations in most $\mathrm{A}_{2} B \mathrm{X}_{4}$ compounds. On the other hand, the form of the soft-phonon branch strongly depends on the force model. It is sufficient to fit the model to the static structure observed at 145 $\mathrm{K}$ instead of the one at room temperature, to provoke a conspicuous softening of the branch. The branch minimum is specially sensitive to some potassium-oxygen interactions. The relative size of the cations plays an essential role in the origin of the incommensurate instability. For comparison the results of a similar analysis for $\mathrm{Cs}_{2} \mathrm{SeO}_{4}$ are presented. In this case, the unstable or "soft" character of the lowest $\Sigma_{2}$ branch disappears.
\end{abstract}

\section{INTRODUCTION}

A considerable number of materials of the type $\mathrm{A}_{2} B X_{4}$ $\left(\mathrm{K}_{2} \mathrm{SeO}_{4}, \mathrm{Rb}_{2} \mathrm{ZnCl}_{4}, \mathrm{Rb}_{2} \mathrm{ZnBr}_{4}, \mathrm{Rb}_{2} \mathrm{CoCl}_{4}, \mathrm{Rb}_{2} \mathrm{CoBr}_{4}\right.$, $\mathrm{K}_{2} \mathrm{ZnCl}_{4}$, etc.) exhibit a common sequence of phase transitions as the temperature is varied. At a certain temperature range they are isomorphous to $\beta-\mathrm{K}_{2} \mathrm{SO}_{4}$, having space group Pnam and a pseudohexagonal axis along the $a$ direction. Below some temperature $T_{i}$, this structure is unstable and the compounds transform into an incommensurately modulated (INC) phase with its wave vector along the $a$ axis (in most cases close to the value $\frac{1}{3} \mathbf{a}^{*}$ ). The symmetry of the primary distorting mode (order parameter) is $\Sigma_{2}$. At lower temperatures, a second phase transition into a commensurate phase takes place and the wave vector locks into a commensurate value (for a comprehensive review see Refs. 1 and 2). Thus, a scheme of the common transition sequence is

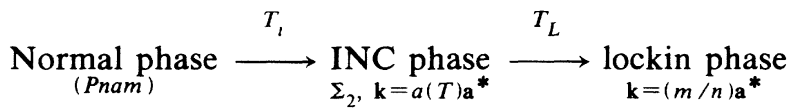

This general scheme is reproduced in all compounds, but some particular features can vary considerably. For instance, $T_{i}$ is $130 \mathrm{~K}$ in $\mathrm{K}_{2} \mathrm{SeO}_{4}$, but $553 \mathrm{~K}$ in $\mathrm{K}_{2} \mathrm{ZnCl}_{4}$. The temperature range of the INC phase changes from $15 \mathrm{~K}$ in $\mathrm{Cs}_{2} \mathrm{CdBr}_{4}$ to $155 \mathrm{~K}$ in $\mathrm{Rb}_{2} \mathrm{ZnBr}_{4}{ }^{2}$ The compounds of $\mathrm{Zn}$ do not exhibit a proper soft-phonon branch, while in $\mathrm{K}_{2} \mathrm{SeO}_{4}$ the existence of a $\Sigma_{2}$ soft mode was clearly observed. ${ }^{2}{ }^{3}$ In the $\mathrm{Zn}$ compounds, the tetrahedral groups $B X_{4}$ are disordered in the normal phase with two equivalent positions related by a mirror plane. ${ }^{4}$ In contrast, $x$-ray structure determinations of the normal phase of $\mathrm{K}_{2} \mathrm{SeO}_{4}$ have been successful using a nondisordered model. ${ }^{5}$ In this last case, however, large thermal ellipsoids indicate strong librations of the ionic groups and recently some spectroscopic anomalies in this compound have been interpreted in terms of a disordered model. ${ }^{6,7}$

Despite these differences, it is reasonable to assume that the normal-INC instability observed in all these compounds has essentially a common origin. A comparison of the eigenvectors describing the modulation or distorting mode in their INC phase supports this viewpoint. This eigenvector is shown in Table I for several $A_{2} B X_{4}$ materials. The atomic modulations corresponding to atoms belonging to the $B X_{4}$ tetrahedral groups have been adjusted to a rigid-body model and in the table only the fitted Fourier amplitudes of the symmetry-allowed rotations and displacements of these groups are listed. A strong similarity among the different distortions is clearly observed (see also Ref. 8 for a comparison considering all atoms independently). Only in the case of $\mathrm{K}_{2} \mathrm{SeO}_{4}$, a significative difference in the relative weight of the amplitudes of the $B X_{4}$ rotations along the $a$ and $b$ axes with respect to the other compounds is detected. The INC modulation is therefore not very sensitive to the details of 
TABLE I. Polarization vector of the modes describing the INC modulation of some $A_{2} B X_{4}$ compounds. $F$ indicates the degree of fitting to rigid-body displacements. For each ion or ionic group in the asymmetric unit, the amplitude of the displacements (relative units $\times 10^{4}$ ) and/or rotations (deg) together with the corresponding phase $(/ 2 \pi)$ are given.

\begin{tabular}{lccccrr}
\hline \hline & & $\mathrm{K}_{2} \mathrm{SeO}_{4}$ & $\mathrm{Rb}_{2} \mathrm{ZnCl}_{4}$ & $\mathrm{Rb}_{2} \mathrm{ZnBr}_{4}$ & $\mathrm{~K}_{2} \mathrm{ZnCl}_{4}$ & $\mathrm{Cs}_{2} \mathrm{CdBr}_{4}$ \\
\hline$A(\alpha)$ & $T_{z}$ & $69 / 0.102$ & $69 / 0.101$ & $143 / 0.110$ & $377 / 0.097$ & $83 / 0.067$ \\
$A(\beta)$ & $T_{z}$ & $92 / 0.321$ & $80 / 0.231$ & $147 / 0.238$ & $430 / 0.287$ & $84 / 0.078$ \\
$B X_{4}$ & $T_{z}$ & $58 / 0.825$ & $64 / 0.771$ & $118 / 0.778$ & $262 / 0.828$ & $43 / 0.695$ \\
& $R_{x}$ & $2.27 / 0.036$ & $5.30 / 0.024$ & $9.66 / 0.025$ & $14.49 / 0.013$ & $6.67 / 0.016$ \\
& $R_{y}$ & $3.68 / 0.643$ & $2.55 / 0.654$ & $4.27 / 0.654$ & $9.71 / 0.626$ & $1.13 / 0.695$ \\
$F$ & & $92 \%$ & $89 \%$ & $92 \%$ & $73 \%$ & $84 \%$ \\
\hline \hline
\end{tabular}

the interatomic interactions and seems to originate essentially in some of the common structural features of all these materials. This type of conclusion is consistent with some recent arguments, which introduce the concept of "latent symmetry" (a certain imperfect symmetry of the structure) as the ultimate cause of the appearance of the INC modulation. ${ }^{9}$

However, a clear understanding of the microscopic mechanism of the INC instability and its connection with the static structural properties of these structures is still missing. Apart from the phenomenological treatments in the frame of Landau theory, only a few works have attempted to explain the INC instability from a microscopic viewpoint. Iizumi et al. ${ }^{3}$ suggest that the instability in $\mathrm{K}_{2} \mathrm{SeO}_{4}$ is the result of a competition between the first-, second-, and third-neighbor interlayer effective interactions in a one-dimensional local-mode layer model along the $x$ direction, with the effective cell corresponding to half the $a$ parameter. This model implies the consideration that the local mode has stronger effective interactions with atoms at distances of about $10 \AA$ than with atoms at distances smaller than $3.8 \AA$. This strong assumption has been no obstacle for the model to be widely accepted and the argument has been reproduced in many subsequent works. More sophisticated models in the frame of mean-field approximation reduce the interactions to first- and second-neighbor layers. ${ }^{10}$ Other studies propose the anharmonic polarizability of the $X$ ions as the origin of the structural instability (at least in the case of $\left.\mathrm{K}_{2} \mathrm{SeO}_{4}\right){ }^{11}$ Finally, some recently reported investigations on the stability of these materials, including the introduction of the mentioned concept of latent symmetry, have been achieved through the analysis of the harmonic lattice dynamics of rigid-ion models with interatomic central forces. ${ }^{9,12,13}$ These models seem to be sufficient to reproduce the essential features of the energetic and vibrational properties of these materials.

In particular, the lattice dynamics of potassium selenate has been studied from a theoretical point of view by Haque and Hardy. ${ }^{12}$ Using a rigid-ion model with aprioristic values for the ionic charges, these authors derived the theoretical phonon branches along the $\Sigma$ line. The necessary parameters for the repulsive pairwise potentials were determined analytically using the set of equations resulting from the equilibrium condition of the structure observed at room temperature. For the pairs oxygen-oxygen and oxygen-selenium no functional form was assumed and only the necessary first and second derivatives at the distances corresponding to the actual bonds were determined. The resulting positive sign for the first derivative of the repulsive force between some of the atoms pairs was, however, incoherent with its nominal repulsive character. For the pairs oxygen-potassium a Born-Mayer potential was considered with some ad hoc modifications for its second derivatives. The internal degrees of freedom of the selenate groups were included in the analysis. The obtained higher-frequency external modes deviated systematically to higher values than those observed experimentally. The model, however, was able to reproduce the existence of the soft-phonon branch by varying the second derivative of the repulsive force for a specific pair of oxygen atoms, and the calculated softmode eigenvector agreed quantitatively with the experimental data. ${ }^{12,14}$ The other simulations of the lattice dynamics of $A_{2} B X_{4}$ compounds have been performed using "ab initio" potentials. 9,13

In the present paper a new analysis of the lattice dynamics of $\mathrm{K}_{2} \mathrm{SeO}_{4}$ and its relation with the lattice INC instability is reported using a semiempirical rigid-ion model. We center our study on the compound $\mathrm{K}_{2} \mathrm{SeO}_{4}$, as it is one of the best known from the experimental point of view, but the main aim is to clarify the lattice-dynamical origin of the structural instability leading to the INC phase in the whole $A_{2} B X_{4}$ family.

One of the questions to elucidate refers to the abovementioned concept of latent symmetry. This concept has been introduced in lattice-dynamics studies that simultaneously stress the necessity of $a b$ initio specific interatomic potential for reproducing the INC instability. However, if the INC instability can be traced back to rather general pseudosymmetric features of the structure of the normal phase, it would be more reasonable to expect that the lattice dynamics of any model of interatomic forces compatible with the observed structure should exhibit "pathological" dynamical characteristics leading to the mentioned structural instability and the observed INC modulation. To check this point, the rigid-ion model used in the present study only considers semiempirical interatomic potentials. In addition, we expect that the use of a fitted semiempirical model can lead to more realistic results than first-principles models, as the first ones can absorb at least partially crystal-field effects.

In the same line of simplifying the model without losing the essential structural and dynamical features of the 
material, a second important characteristic of the present analysis is that the $B X_{4}$ groups are considered rigid units. This contrasts with previous vibrational studies that have included the interactions between the atoms inside the tetrahedral groups. $9,12,13$ The internal interaction $B$ - $X$ has been even considered of some relevance in the transition mechanism in the case of $\mathrm{Rb}_{2} \mathrm{ZnCl}_{4} \cdot{ }^{9}$ As can be seen in Table $\mathrm{I}$, the tetrahedral groups $B X_{4}$ are approximately rigid in the normal-incommensurate transition in all the compounds. Therefore, their internal degrees of freedom are expected to be essentially inert and irrelevant in the transition. Only the external vibrations should play a major role in the lattice-dynamical model of the INC instability. By this means, the number of parameters of the interatomic force model to be fitted is reduced to two or five depending on the model used (see next section), which compares favorably with the 14 parameters for the static equilibrium and more than 20 additional parameters fitted using experimental vibrational data, which are considered in Ref. 12.

The model parameters for the interatomic forces to be used in the dynamical analysis were optimized using the condition that the resulting atomic equilibrium configuration should be a good approximation of the observed structure (see Table II). As atomic equilibrium configuration, we mean the atomic positions and the unit-cell parameters, which minimize the potential energy with respect to any structural distortion compatible with the fixed orthorhombic Pnam symmetry. Consequently, for this configuration the derivatives of the total potential energy with respect to any structural variable, which is homogeneous in each cell and compatible with the Pnam symmetry, is zero. This requirement is necessary but obviously not sufficient to ensure that the structure is stable with respect to modulated distortions or symmetrybreaking modes in general. It is, however, enough to determine the values of the energetic parameters describing the model of interatomic forces.

Once the model is chosen under the above conditions, the external phonon dispersion branches in the harmonic approximation have been calculated for the different models. Eventual instabilities of the structure with respect to symmetry-breaking modes will be accompanied by the existence of imaginary frequencies in one or more dispersion branches. We analyze thoroughly the eventual presence of these unstable modes as they can be considered the main factor leading to a thermal structural instability of the Pnam structure. The eigenvector of the most unstable lattice mode can then be compared with the observed soft mode in the real system. By varying specific interatomic interactions we also investigate the essential factors that are responsible for the INC instability and the degree of sensitivity of the eigenvector of the modulation to the interaction model. For comparison, we present the results of a similar analysis for $\mathrm{Cs}_{2} \mathrm{SeO}_{4}$, which demonstrate the crucial role played by the effective volume of the $A$ cations on the origin of the INC instability.

Obviously, the present analysis, although microscopic, adopts a mechanical picture of the material. Thermal effects are only considered from a phenomenological point of view, in the sense that we assume that thermal renormalization of the mode frequencies can be strong enough to stabilize at high enough temperatures the set of modes that are unstable in a mechanical picture. The model of adjustable interatomic forces is partially adapted to the thermal effects, since it is the structure at a finite temperature that is taken as the observed structure in the fitting process. In this sense, it can be considered a set of thermal effective forces.

\section{INTERATOMIC FORCES}

We expect that the presence of the INC instability is essentially independent of the details of the interatomic forces. Therefore, the model is strongly simplified. In particular, only Coulomb rigid-ion and Born-Mayer repulsive forces are introduced. In contrast with previous studies, the $\mathrm{SeO}_{4}$ groups are taken as rigid units in the room-temperature configuration. The interatomic potentials $V\left(r_{i j}\right)$ are taken as $V_{c}\left(r_{i j}\right)+V_{\text {rep }}\left(r_{i j}\right)$, with

$$
V_{c}\left(r_{i j}\right)=Q_{i} Q_{j} / r_{i j}
$$

and

$$
V_{\text {rep }}\left(r_{i j}\right)=A_{i j} \exp \left(-B_{i j} r_{i j}\right)
$$

TABLE II. Experimental structure of potassium selenate at room temperature and at $145 \mathrm{~K}$, and cesium selenate at room temperature. The lattice constants are given in $\AA$, and the atomic positions in relative units $\left(\times 10^{4}\right)$. The data are taken from Refs. 5 , 17 ,

\begin{tabular}{|c|c|c|c|c|c|c|c|c|c|c|}
\hline & \multicolumn{3}{|c|}{$\mathrm{K}_{2} \mathrm{SeO}_{4}(293 \mathrm{~K})$} & \multicolumn{3}{|c|}{$\mathrm{K}_{2} \mathrm{SeO}_{4}(145 \mathrm{~K})$} & \multicolumn{4}{|c|}{$\mathrm{Cs}_{2} \mathrm{SeO}_{4}(293 \mathrm{~K})$} \\
\hline$a$ & & & 7.661 & & 7.5 & & & & & \\
\hline$b$ & & & 10.466 & & 10.3 & & & 11. & & \\
\hline \multirow[t]{2}{*}{$c$} & & & 6.003 & & 5. & & & & & \\
\hline & $x$ & $y$ & $z$ & $x$ & $y$ & $z$ & & $x$ & $y$ & $z$ \\
\hline $\mathrm{Se}$ & 2242 & 4200 & 2500 & 2228 & 4195 & 2500 & $\mathrm{Se}$ & 2348 & 4188 & 2500 \\
\hline $\mathbf{K}(\alpha)$ & 1705 & 843 & 2500 & 1670 & 823 & 2500 & $\operatorname{Cs}(\alpha)$ & 1768 & 900 & 2500 \\
\hline $\mathbf{K}(\beta)$ & -57 & 7095 & 2500 & -35 & 7076 & 2500 & $\operatorname{Cs}(\beta)$ & -105 & 7061 & 2500 \\
\hline $\mathrm{O}(1)$ & 2931 & 3471 & 271 & 2918 & 3440 & 274 & $\mathrm{O}(1)$ & 3032 & 3525 & 418 \\
\hline $\mathbf{O}(2)$ & 3024 & 5644 & 2500 & 3083 & 5638 & 2500 & $\mathrm{O}(2)$ & 3022 & 5546 & 2500 \\
\hline $\mathrm{O}(3)$ & 126 & 4251 & 2500 & 102 & 4274 & 2500 & $\mathrm{O}(3)$ & 392 & 4178 & 2500 \\
\hline
\end{tabular}
and 23 , respectively. 
As the $\mathrm{SeO}_{4}$ tetrahedra are considered rigid, their internal interatomic interactions are not included. The repulsive forces between the selenium atoms and atoms external to the tetrahedra can also be reasonably neglected. Obviously the oxygen-oxygen interaction is considered only between atoms belonging to different $\mathrm{SeO}_{4}$ groups. The repulsive interaction $\mathrm{K}-\mathrm{K}$ was included in preliminary calculations, but its influence on the results was very weak, as they involve comparatively long interatomic distances. Consequently, energetic contributions of the K-K repulsive forces were neglected in further calculations.

The potassium ions were taken to be fully ionized, $Q_{\mathrm{K}}=+1 e$. Thus, the charge of the oxygen ion was related to the one of the selenium ions by the neutrality condition $Q_{\mathrm{Se}}+4 Q_{\mathrm{O}}=-2 e$. As repulsive parameters $A_{\mathrm{O}-\mathrm{O}}$ and $B_{\mathrm{O}-\mathrm{O}}$, we took those proposed in Ref. 15 ( $A_{\text {O-O }}=55 \times 10^{3} \mathrm{kcal} / \mathrm{mol}, B_{\text {O-O }}=3.96 \AA^{-1}$ ). The value of $B_{\mathrm{K}-\mathrm{O}}$ was fixed at $3.76 \AA^{-1}$, obtained from the values of $B_{\mathrm{K}-\mathrm{K}}$ and $B_{\mathrm{O}-\mathrm{O}}$ and the approximate empirical rule $B_{i j}=\left(B_{i i}+B_{j j}\right) / 2$. In this relation, the value for $B_{\mathrm{K}-\mathrm{K}}$ was taken to be $3.56 \AA^{-1}$. This value results from considering the values of $B_{\mathrm{K}-\mathrm{F}}$ and $B_{\mathrm{F}-\mathrm{F}}$ given in Ref. 16 and the empirical rule mentioned above.

It is well known that the parameters $B_{i j}$ are strongly correlated with the $A_{i j}$ values, so that similar potentials can be described by different sets of both parameters. Therefore the values given $a$ priori to the $B_{i j}$ parameters are not a determinant restriction in the present study.

The remaining energetic parameters $\left(Q_{\mathrm{Se}}, A_{\mathrm{K}-\mathrm{O}}\right)$ were adjusted, as explained in the preceding section, using the condition that the resulting atomic equilibrium configuration should be a good approximation of the observed structure either at room temperature, ${ }^{5}$ or at $145 \mathrm{~K}$ just above $T_{i}$ (Ref. 17) (see Table II). We used for this process a program based on a SIMPLEX algorithm ${ }^{18}$ that searched the energetic parameters minimizing the structural differences between the corresponding "equilibrium configuration" and the observed structure. The "equilibrium configuration" was calculated at each step of the SIMPLEX process by "relaxing" a convenient initial structure (in most cases the experimental one at room temperature). The program WMIN working in mode 1
(Newton-Raphson energy minimization mode) ${ }^{19}$ was used for this purpose. The numerical factor that was in fact minimized and quantitatively measured the difference between the observed and relaxed structure is defined by

$$
\begin{aligned}
R=(1 / N) & \sum_{i=1,3}\left[\left(\Delta a_{i} / a_{i}^{0}\right) 100\right]^{2} \\
& \left.+\sum_{j} \sum_{i}\left[\Delta x_{j, i} a_{i}^{0}(10)\right]^{2}\right]^{1 / 2},
\end{aligned}
$$

where $\Delta a_{i}$ and $\Delta x_{j, i}$ are, respectively, the deviations of the lattice constants and of the atomic fractional positions from the observed values, while $a_{i}^{0}$ are the experimental lattice constants in $\AA$. The sum in $j$ extends to all the atoms in the asymmetric unit, and the sum in $i$ is restricted to those components that are not fixed by symmetry requirements. $N$ is the number of terms in the sum. According to its definition, this parameter is equal to one in the hypothetical case that all unit-cell parameters deviate $1 \%$ and all the atomic coordinates differ in $0.1 \AA$ from the experimental observation. This order of magnitude for the discrepancies between the simulated structural model and the real observation is normally considered acceptable in this type of study. ${ }^{20}$ In the present study, the factor $R$ attained values of the order of $0.1-0.3$. It can be noted that these values are 1 order of magnitude smaller than the one $(R=1.42)$ corresponding to the relaxed structure obtained for $\mathrm{Rb}_{2} \mathrm{ZnCl}_{4}$ in Ref. 9 using an $a b$-initio force model.

The significance of the quantitative value of $R$ was investigated by determining its value for the structural difference between two relaxed structures corresponding to force models differing only in the charge values an amount $\Delta Q_{\mathrm{Se}}=0.1 e\left(\Delta Q_{\mathrm{O}}=0.025 e\right)$. Typically $R$ took values between 0.04 and 0.09 .

The best fit was finally obtained for $Q_{\mathrm{Se}}=+1.30 e$ $\left(Q_{\mathrm{O}}=-0.825 e\right)$ and $A_{\mathrm{K}-O}=97.7 \times 10^{3} \mathrm{Kcal} / \mathrm{mol}$ with $R=0.159$. The relaxed structure is presented in Table III. In the following, we shall call the resulting model of interatomic forces, model 1 . The whole set of model pa-

TABLE III. Relaxed structure of potassium selenate for model 1, model 2 (293 K), and model 2 (145 K), and of cesium selenate for model 1. The lattice constants are given in $\AA$, and the atomic positions in relative units $\left(\times 10^{4}\right)$.

\begin{tabular}{|c|c|c|c|c|c|c|c|c|c|c|c|c|c|c|}
\hline & $x$ & $y$ & $z$ & $x$ & $y$ & & & $x$ & $y$ & $z$ & & $x$ & $y$ & $z$ \\
\hline $\mathrm{Se}$ & 2333 & 4181 & 2500 & 2319 & 4190 & 2500 & & 2291 & 4191 & 2500 & $\mathrm{Se}$ & 2363 & 4179 & 2500 \\
\hline $\mathbf{K}(\alpha)$ & 1644 & 856 & 2500 & 1657 & 887 & & 2500 & 1629 & 879 & 2500 & $\operatorname{Cs}(\alpha)$ & 1684 & 895 & 2500 \\
\hline $\mathbf{K}(\beta)$ & -76 & 6963 & 2500 & -95 & 7023 & 2500 & & -68 & 7022 & 2500 & $\operatorname{Cs}(\beta)$ & -111 & 6971 & 2500 \\
\hline$O(1)$ & 3006 & 3440 & 297 & 3030 & 3467 & & 296 & 2997 & 3440 & 293 & $\mathbf{O}(1)$ & 3036 & 3506 & 437 \\
\hline $\mathrm{O}(2)$ & 3156 & 5620 & 2500 & 3071 & 5652 & & 2500 & 3127 & 5648 & 2500 & $\mathbf{O}(2)$ & 3065 & 5537 & 2500 \\
\hline $\mathrm{O}(3)$ & 207 & 4261 & 2500 & 189 & 4214 & 2500 & & 152 & 4252 & 2500 & $\mathrm{O}(3)$ & 401 & 4191 & 2500 \\
\hline
\end{tabular}

\begin{tabular}{lcccc}
\hline & $\mathrm{K}_{2} \mathrm{SeO}_{4}$ model 1 & $\mathrm{K}_{2} \mathrm{SeO}_{4}$ model 2 $(293 \mathrm{~K})$ & $\mathrm{K}_{2} \mathrm{SeO}_{4}$ model 2 (145 K) & $\mathrm{Cs}_{2} \mathrm{SeO}_{4} \mathrm{model}^{1}$ \\
\hline$a$ & 7.662 & 7.611 & 7.556 & 8.352 \\
$b$ & 10.426 & 10.413 & 10.335 & 11.219 \\
$c$ & 6.074 & 6.070 & 6.028 & 6.496
\end{tabular}




\begin{tabular}{|c|c|c|c|c|c|}
\hline & $\begin{array}{l}\text { Model } 1 \\
\left(\mathrm{~K}_{2} \mathrm{SeO}_{4}\right)\end{array}$ & $\begin{array}{c}\text { Model } 2 \\
\left(\mathrm{~K}_{2} \mathrm{SeO}_{4}-293 \mathrm{~K}\right) \\
\end{array}$ & $\begin{array}{c}\text { Model } 2 \\
\left(\mathrm{~K}_{2} \mathrm{SeO}_{4}-145 \mathrm{~K}\right) \\
\end{array}$ & & $\begin{array}{l}\text { Model } 1 \\
\left(\mathrm{Cs}_{2} \mathrm{SeO}_{4}\right) \\
\end{array}$ \\
\hline$R$ & 0.159 & 0.132 & 0.105 & & 0.109 \\
\hline$Q_{\mathrm{Se}}(e)$ & 1.30 & 1.34 & 1.34 & & 1.44 \\
\hline$Q_{\mathrm{O}}(e)$ & -0.825 & -0.835 & -0.835 & & -0.86 \\
\hline$B_{\mathrm{K}-\mathrm{O}}\left(\AA^{-1}\right)$ & 3.76 & 3.76 & 3.76 & $\boldsymbol{B}_{\mathrm{Cs}-\mathrm{O}}$ & 3.50 \\
\hline$A_{\mathrm{K}(\alpha)-\mathrm{O}(1-2)}\left(10^{3} \mathrm{Kcal} / \mathrm{mol}\right)$ & & 114.7 & 106.6 & & \\
\hline$A_{\mathrm{K}(\alpha)-\mathrm{O}(3)}$ & & 102.6 & 97.4 & & \\
\hline$A_{\mathrm{K}(\beta)-\mathrm{O}(1-2)}$ & 97.7 & 83.3 & 75.4 & $A_{\mathrm{Cs}-\mathrm{O}}$ & 122.5 \\
\hline$A_{\mathrm{K}(\beta)-O(3)}$ & & 118.5 & 100.0 & & \\
\hline
\end{tabular}

rameters is listed in Table IV. A change of the selenium charge of $0.1 e$ did not change significantly the quality of the adjustment. Fixing $Q_{\mathrm{Se}}$ to $+1.6 e$ is, however, enough to double the $R$ value. Any attempt to fix the charge of the selenium and oxygens to higher values failed. The WMIN program in mode 1 was unable in these cases to relax properly from the experimental structure into an equilibrium configuration, indicating that the latter does not exist or differs considerably from the experimental one. This result contrasts with the analysis of the same compound in Ref. 12, where the charge of the selenium ion was fixed to $+2 e\left(Q_{\mathrm{O}}=-1 e\right)$. On the other hand, a recent molecular dynamics study of the related compound $\mathrm{LiKSO}_{4}$ considered an oxygen charge even smaller than those obtained in the present study. ${ }^{21}$

The results of the lattice-dynamics analysis of force model 1 , described in the next section, led us subsequently to increase the complexity of the force model by assuming specific repulsive interactions for the pairs $\mathrm{K}-\mathrm{O}$ depending on the crystallographic type of potassium or oxygen considered. There are two types of inequivalent potassium atoms in the structure that occupy very different positions from a topological point of view (see Fig. 1). The $\mathbf{K}(\beta)$ occupy a cavity of approximate hexagonal symmetry along the $a$ axis and is surrounded by six tetrahedra. Its separation from the oxygen atoms $\mathrm{O}(1)$ and $\mathrm{O}(2)$, on the face of the tetrahedra perpendicular to the $a$ axis varies from 2.74 to $2.82 \AA$, while the shortest distance to an oxygen on the other vertex, $\mathrm{O}(3)$, is already $2.97 \AA$. In contrast, the $\mathrm{K}(\alpha)$ are situated approximately on some of the vertices of the hexagonal cavities (see Fig. 1), such that their shortest contact corresponds to an oxy-

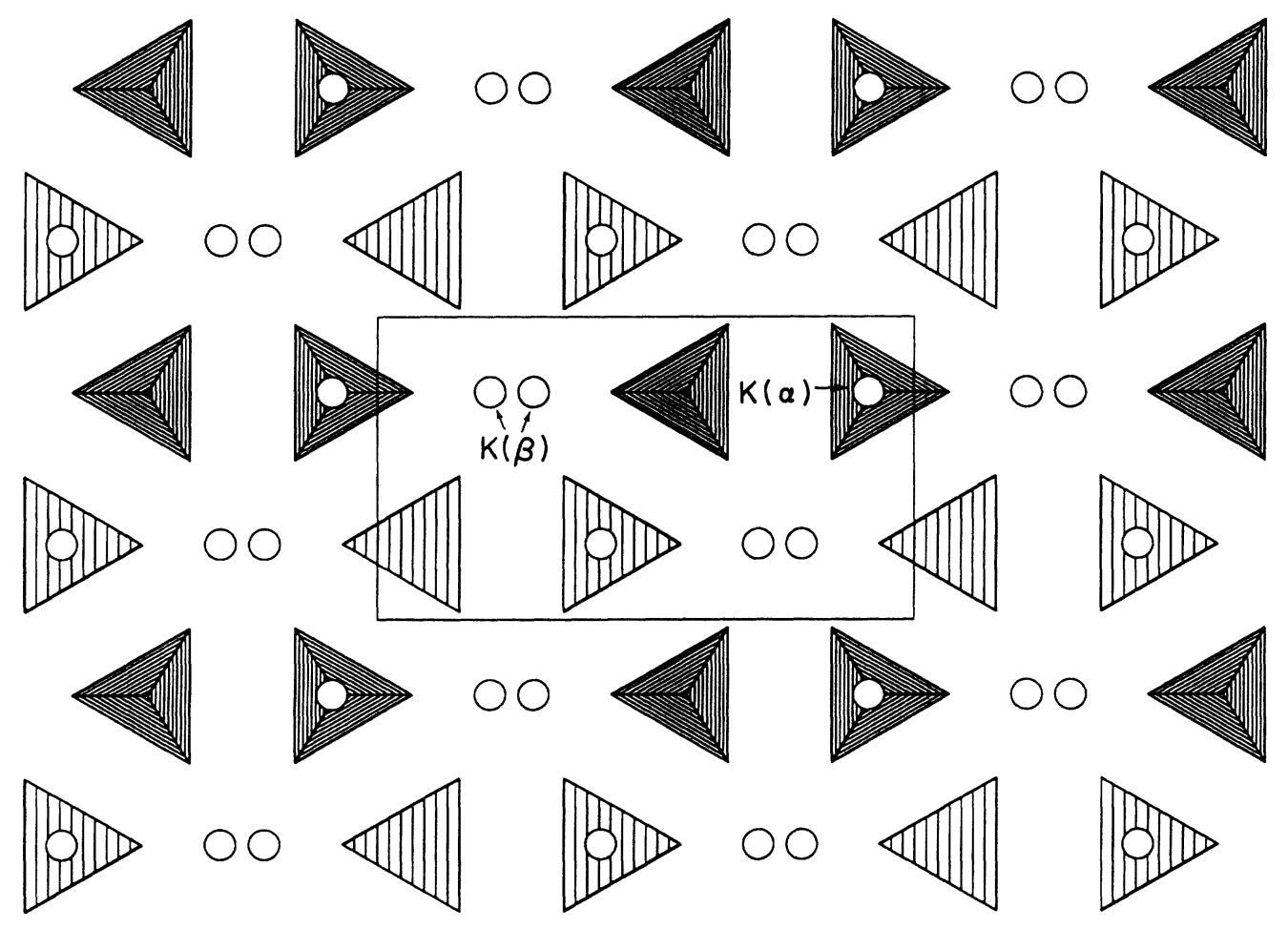

FIG. 1. Projection along the $a$ axis of the structure of $\mathrm{K}_{2} \mathrm{SeO}_{4}$ at room temperature showing the two kinds of inequivalent potassium atoms. 
gen $\mathrm{O}(3)$ at $2.62 \AA$, while the next ones are already at distances varying from 3.00 to $3.26 \AA$ and correspond to oxygens of the type $\mathrm{O}(1)$ or $\mathrm{O}(2)$. It is well known that effective repulsive potentials can vary considerably when different ranges of atomic distances are considered, so that they are described by different Born-Mayer potentials. Also, ab-initio interactions like Gordon-Kim potentials are given by different analytical expressions for different distance ranges. ${ }^{11}$ It is therefore reasonable to assume a set of four different Born-Mayer K-O potentials, if we take into account the very different interatomic distances relevant in each case. Accordingly, we split the initial unique K-O Born-Mayer potential into four different potentials depending on the atoms considered: $\mathrm{K}(\alpha)-\mathrm{O}(1-2), \mathrm{K}(\alpha)-\mathrm{O}(3), \mathrm{K}(\beta)-\mathrm{O}(1-2)$, and $\mathrm{K}(\beta)-\mathrm{O}(3)$.

The number of adjustable parameters in this generalized model increases to five: the effective charge of selenium and the four parameters $A_{\mathrm{K}-\mathrm{O}}$ (the parameter $B_{\mathrm{K}-\mathrm{O}}$ is kept the same for the four different pairs and has the value given above). An optimal set of these parameters was determined by means of a fitting process analogous to the preceding one. The process converged to a value of the Se charge of $+1.6 e\left(Q_{\mathrm{O}}=-0.9 e\right)$ with a fitting factor $R=0.109$. However lower values such as $+1.3 e$ did not deteriorate the adjustment significantly and $R$ increased only up to $R=0.136$. The subsequent normal-mode analysis indicated that with Se charges as high as $+1.6 e$, the system was unstable along the $\Sigma$ line. Therefore the Se charge was fixed to a lower value $(+1.34 e)$ and only the parameters $A_{\mathrm{K}-\mathrm{O}}$ were optimized. The resulting model, henceforth referred to as model 2 (293 K), is listed in Table IV. In Table III, the corresponding Pnam relaxed structure is also presented. As in the previous case, when higher values than $1.6 e$ were considered no proper Pnam relaxed structure close to the experimental one could be found.

The resulting configurational energy of the equilibrium structure $[-416.39 \mathrm{Kcal} / \mathrm{mol}$ for model $1,-416.34$ $\mathrm{Kcal} / \mathrm{mol}$ for model $2(293 \mathrm{~K})$ ] is close to the experimental cohesion energy $(-413.76 \mathrm{Kcal} / \mathrm{mol}){ }^{22}$

A third model [model $2(145 \mathrm{~K})$ in Table IV] was considered using as observed equilibrium configuration the structure determined at $145 \mathrm{~K}$ (Ref. 17) (see Table II) and maintaining $Q_{\mathrm{Se}}=+1.34 e$. Essentially, as a consequence of the thermal contraction, a decrease of the effective $\mathrm{K}$ $O$ repulsive interactions with respect to the roomtemperature model is obtained.

Finally, for $\mathrm{Cs}_{2} \mathrm{SeO}_{4}$, a similar process was followed. A simple model with a single $\mathrm{Cs}-\mathrm{O}$ interaction analogous to model 1 for $\mathrm{K}_{2} \mathrm{SeO}_{4}$ was fitted following the same procedure. As observed structure we considered the recently determined structure at room temperature ${ }^{23}$ (see Table II). The O-O interaction was kept the same as in potassium selenate, while the value of $B_{\text {Cs-O }}$ was fixed to 3.50 $\AA^{-1}$. The results are also given in Tables III and IV. The final optimized values of $Q_{\mathrm{Se}}$ and $A_{\mathrm{Cs}-\mathrm{O}}$ were $+1.44 e$ and $122.5 \times 10^{3} \mathrm{Kcal} / \mathrm{mol}$, respectively, with $R=0.109$. Thus, the repulsive interaction $A-O$ increases considerably with respect to potassium selenate, in accordance with the corresponding increase of the unit-cell volume and the effective atomic size of the $A$ atoms.

\section{LATTICE DYNAMICS}

\section{A. Introduction}

Using the same notation for the irreducible representations as in Ref. 3, the symmetry decomposition at the center of the Brillouin zone of the external normal modes is

$7 A_{g}+7 B_{1 g}+5 B_{2 g}+5 B_{3 g}+5 A_{u}+5 B_{1 u}+7 B_{2 u}+7 B_{3 u}$.

Along the $\Sigma$ line $\left(q=\alpha a^{*}\right)$ the decomposition becomes

$$
14 \Sigma_{1}+10 \Sigma_{2}+10 \Sigma_{3}+14 \Sigma_{4}
$$

and at the point $X\left(\mathbf{q}=\frac{1}{2} \mathbf{a}^{*}\right)$ is

$$
14 X_{1}+10 X_{2} \text {. }
$$

The notation for the irreducible representations at the center of the Brillouin coincides with the one of Refs. 6 and 7.

The number of branches $\Sigma_{2}$ and $\Sigma_{3}$ is the same, and the same happens for $\Sigma_{1}$ and $\Sigma_{4}$. The compatibility relations ${ }^{3}$ are such that each pair of them are degenerate at the point $X$. Therefore, in an extended zone scheme each pair of branches $\Sigma_{2}-\Sigma_{3}$, or $\Sigma_{1}-\Sigma_{4}$, reduces to a single branch, and the total number of branches reduces to 24 . In the following, we will use this extended zone scheme for the graphical representation of the phonon branches.

Iizumi et $a l .^{3}$ determined by means of inelastic neutron scattering the existence of a soft-mode $\Sigma_{2}$ branch connected with a $\Sigma_{3}$ acoustic branch in $\mathrm{K}_{2} \mathrm{SeO}_{4}$. The instability of this $\Sigma_{2}$ branch at a point close to $0.31 \mathrm{a}^{*}$ takes place at a temperature consistent with the known transition temperature $T_{t}=130 \mathrm{~K}$.

Several Raman and infrared spectroscopic measurements of $\mathrm{K}_{2} \mathrm{SeO}_{4}$ have been reported in the last few years. $6,7,24-28$ The number of internal modes detected by this means in the normal phase differs from those corresponding to the usual structural model. As a possible cause, it has been suggested that the selenate tetrahedra have some kind of weak disorder, which cannot be detected by $x$-ray methods. ${ }^{6,7}$ On the other hand, the external Raman and infrared-active modes $\left(A_{g}, B_{1 g}, B_{2 g}\right.$, $B_{3 g}$ and $B_{1 u}, B_{2 u}, B_{3 u}$, respectively) have been identified and measured without much problem in the frame of the symmetry analysis above. ${ }^{6,7}$ The experimental data reported in Ref. 6 will be used for comparison with the present simulation. A significant fact can be deduced from these studies: apparently, the soft $\Sigma_{2}$ branch becomes an optically inactive $A_{u}$ mode at the center of the Brillouin zone. This can be derived indirectly from the knowledge that a $\Sigma_{2}$ branch is only compatible with $A_{u}$ or $B_{3 g}$ symmetries at the zone center, and the lowest $B_{3 g}$ measured Raman frequency is at the range of $45 \mathrm{~cm}^{-1}$, while from the neutron data of Iizumi et al, ${ }^{3}$ the end of the soft $\Sigma_{2}$ branch has values lower than $25 \mathrm{~cm}^{-1}$.

\section{B. Phonon branches calculation}

The dispersion curves were calculated under the harmonic approximation using a general program, which al- 


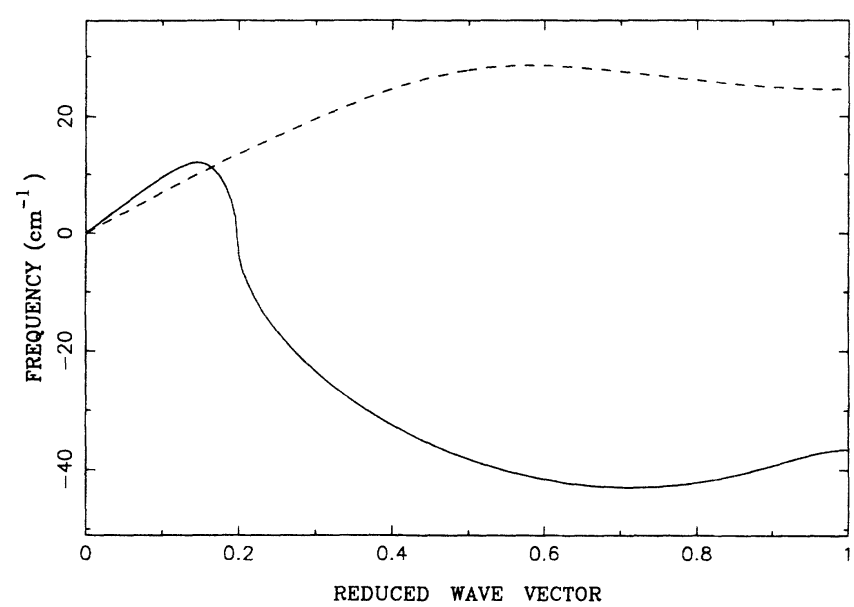

FIG. 2. Calculated dispersion curves plotted in the extended zone scheme corresponding to the model 1 of $\mathrm{K}_{2} \mathrm{SeO}_{4}$ (solid line) and $\mathrm{Cs}_{2} \mathrm{SeO}_{4}$ (dashed line). For imaginary values of the frequency, $-|\omega|$ is represented.

lows us to consider rigid molecular units and therefore can restrict the calculations to the external modes. The external Born-von Karman formalism, ${ }^{29}$ in which the force constants are obtained in terms of molecular rotations and translations, is used. The dynamical matrix is built up using the method of Pawley ${ }^{30}$ where, using a pairwise potential model, force constants are calculated analytically as a function of rotational and translational coordinates. "Self-terms" or force constants relating two displacements of the same molecular unit are obtained from the crystal translational and rotational invariance conditions. ${ }^{29}$

Short-range force constants were calculated for molecular pairs inside a cutoff radius distance $(8 \AA)$. For Coulombic interactions however, given their long-range character, the Ewald convergence method was used. ${ }^{29,31}$

\section{Results}

\section{Model 1}

This model is unstable for vibrations along the $\Sigma$ line, namely, an unstable dispersion branch of $\Sigma_{2}-\Sigma_{3}$ symmetry appears as shown in Fig. 2. No other branch along this line takes imaginary values. A clear minimum with a rather large depth $(5.32 \mathrm{meV})$ can be observed at a point $q=0.28 \mathbf{a}^{*}$. The mode eigenvector at this minimum listed in Table $\mathrm{V}$ already reproduces most of the characteristics of the experimental soft-mode eigenvector in Table I. The agreement between the phases is especially significant, but also the different atomic amplitudes follow in general terms the experimental pattern. Only a significant deviation to larger values of the amplitude of the selenate rotation around the $x$ axis and a too small selenate $z$ translation can be clearly observed. In contrast with the experimental situation, the $\Sigma_{2}$ mode at the zone center has $B_{3 g}$ symmetry. An increase of the Se charge leads, apart from the changes in the equilibrium structure, to a stronger instability of the structure, with the soft-mode imaginary branch taking larger absolute values.

\section{Model 2(293 K)}

The system is stable with respect to all normal modes. Again the lowest nonacoustic branch along the $\Sigma$ line has $\Sigma_{2}$ symmetry, but in this case the frequencies have real values. This branch (see Fig. 3) is rather flat and presents a weak minimum at $q=0.22 \mathrm{a}^{*}$. The mode eigenvector at this point agrees with the experimental one much better than for model 1 (see Tables I and V). The previous discrepancies observed in the amplitudes of $R_{x}$ and $T_{z}$ of the selenate group are corrected, but the deviation of the $T_{z}$ amplitude of the $\mathrm{K}(\alpha)$ atom suffers a slight increase. The phases for the different atoms seem to be weakly dependent on the details of the interactions. They scarcely change between the two models and have an excellent agreement with the experimental values in Table I.

The branch zone center has $A_{u}$ symmetry. However, for larger values of the Se charge (an increase of $0.02 e$ is sufficient) the mode changes to $B_{3 g}$ symmetry. The reason for this extreme sensitivity of the symmetry of the zone-center mode to the details of the interatomic forces is caused by the proximity of another $\Sigma_{2}$ branch around the zone center. The two branches may "interact" and can easily interchange the symmetry of their zone-center modes. The frequency of the lowest $B_{3 g}$ mode is specially sensitive to changes of the ionic charges and strongly decreases for larger charges. At $Q_{\mathrm{Se}}=+1.36 \mathrm{e}$ the interchange of the extremes of the two $\Sigma_{2}$ branches takes place. In addition, this lowering of the $B_{3 g}$ mode causes, by a similar effect, the destabilization (negative values for $C_{44}$ ) of the acoustic branches along the $y$ and $z$ directions corresponding to the shear $e_{y z}$, which has the same symmetry. Further increases of the Se charge leads to lower frequencies all along the lowest $\Sigma_{2}$ branch and strengthens the presence of a minimum at values close to

TABLE V. Eigenvectors of the mode corresponding to the minimum in the soft branch of $\mathrm{K}_{2} \mathrm{SeO}_{4}$ for (a) model 1, (b) model 2 (293 $\mathrm{K})$, (c) model $2(145 \mathrm{~K})$, (d) modified model $2(145 \mathrm{~K})$ changing the $A_{\mathrm{K} \text {-o }}$ parameter for the interaction $\mathrm{K}(\alpha)$-O(1-2). (e) Eigenvector of the mode $\Sigma_{2}$ at $0.31 \mathrm{a}^{*}$ for $\mathrm{Cs}_{2} \mathrm{SeO}_{4}$. The data are given in the same manner as in Table $\mathrm{I}$.

\begin{tabular}{lccrrrr}
\hline \hline & & (a) & (b) & (c) & (d) \\
\hline $\mathrm{K}(\alpha)$ & $T_{z}$ & $61.2 / 0.110$ & $79.6 / 0.135$ & $75.2 / 0.126$ & $72.1 / 0.119$ & $64.6 / 0.118$ \\
$\mathrm{~K}(\beta)$ & $T_{z}$ & $72.8 / 0.318$ & $80.3 / 0.320$ & $83.3 / 0.319$ & $80.8 / 0.320$ & $81.2 / 0.319$ \\
$\mathrm{SeO}_{4}$ & $T_{z}$ & $38.2 / 0.811$ & $68.0 / 0.816$ & $62.6 / 0.820$ & $56.5 / 0.830$ & $45.3 / 0.824$ \\
& $R_{x}$ & $4.06 / 0.012$ & $2.23 /-0.003$ & $2.30 / 0.002$ & $2.70 / 0.008$ & $0.96 /-0.025$ \\
& $R_{y}$ & $3.06 / 0.610$ & $3.58 / 0.621$ & $3.66 / 0.617$ & $3.63 / 0.613$ & $2.46 / 0.614$ \\
\hline \hline
\end{tabular}




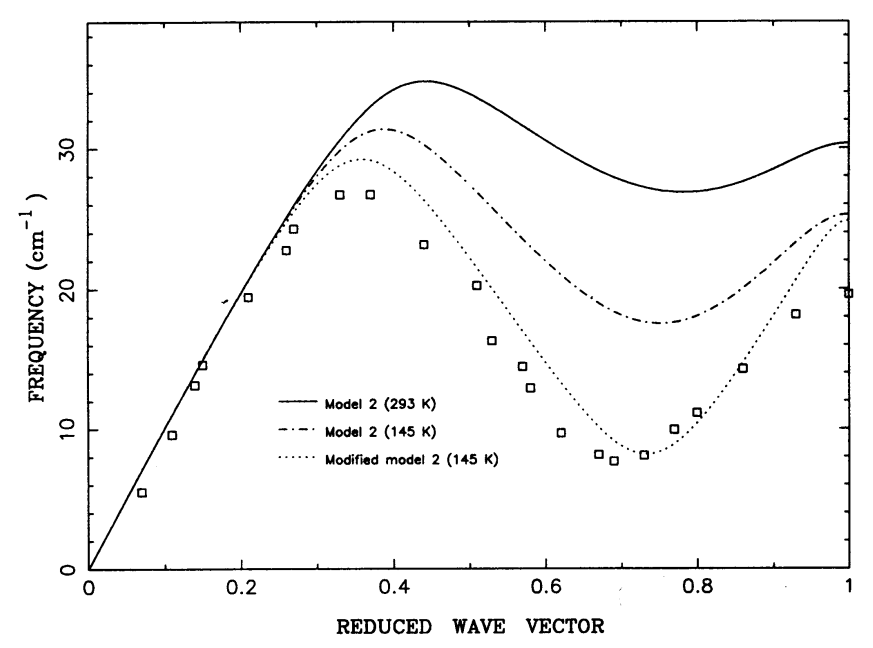

FIG. 3. Calculated dispersion curves of $\mathrm{K}_{2} \mathrm{SeO}_{4}$ plotted in the extended zone scheme for model 2 ( $293 \mathrm{~K}$ ) (solid line), model $2(145 \mathrm{~K})$ (dashed line) and model $2(145 \mathrm{~K})$ decreasing the interaction of the $\mathrm{K}(\alpha)-\mathrm{O}(1-2)$ pairs (point line). The experimental points at $145 \mathrm{~K}$ (Ref. 3 ) are also shown.

$\frac{1}{3} \mathrm{a}^{*}$. For values of $Q_{\mathrm{Se}}$ around $+1.6 e$, which was the optimum value resulting from structural considerations (see Sec. II), the $\Sigma_{2}$ branch is completely unstable, with a similar form to the one of Fig. 2 for model 1. This was the reason to fix the value of $Q_{\mathrm{Se}}$ in this model to $1.34 e$. Thus, the condition of complete stability of the system practically forced the $A_{u}$ symmetry at the zone center of the branch, as observed experimentally.

The elastic constants and Raman frequencies calculated for model 2(293 K) are listed in Tables VI and VII, respectively, where experimental values are also given. ${ }^{32,6}$ A graphical comparison can be seen in Fig. 4. In general, the agreement is within 5-20\%, which is excellent considering that the force model has only been adjusted using static structural data. However, a $B_{1 g}$ mode (the lowest one) strongly deviates from the experimental value. This strong deviation can be due to a symmetry interchange effect similar to the one commented above between the lowest modes of $A_{u}$ and $B_{3 g}$ symmetry. In this case, both $B_{1 g}$ and $B_{2 u}$ modes are compatible with the branches of $\Sigma_{4}$ symmetry. In the simulation, the lowest $\Sigma_{4}$ optic branch ends at the zone center with a $B_{2 u}$ mode (see Fig. 5). This symmetry could be interchanged with the $B_{1 g}$ symmetry of the mode at the end of the next $\Sigma_{4}$ branch if the force model is varied.

For the sake of comparison with previous studies, and also as a reference for eventual subsequent experimental studies, the whole set of external phonon branches with this model is shown in Fig. 5. The density of states corresponding to the external modes is also shown in Fig. 4. The highest external frequencies have values that are significantly smaller than those obtained in Ref. 12, with a better agreement with the experimental data.

The global agreement of the mode frequencies can be confirmed by comparing the resulting specific-heat curve to the experimental results. This is done in Fig. 6, where the effect of the internal frequencies of the selenate groups have been included by adding to the density of states of Fig. 4 four Einstein-type contributions, centered at the approximate experimental values of the selenate internal frequencies. ${ }^{33}$ The experimental specific-heat curve has been taken from Ref. 34. The agreement between experimental and theoretical values is excellent. Only an obvious and expected discrepancy exists close to the transition temperature. The deviation of the experimental values at higher temperatures is due to the usual discrepancy between the experimental specific heat at constant pressure and the theoretical one at constant volume, which is caused by anharmonic effects. This deviation can be fitted to the usual approximate linear temperature dependence.

Thus, model 2 reproduces quantitatively up to an excellent degree many of the known physical properties of potassium selenate. It should be noted that the model was not chosen to fit the experimental dynamical properties and only the condition of mechanical stability of the room-temperature structure was used to adjust the model parameters.

\section{Model 2(145 K)}

As explained in Sec. II, this model was obtained as a modification of model $2(293 \mathrm{~K})$, using as observed structure the one determined at $145 \mathrm{~K}$ (Ref. 17) (see Table II) to adjust the model parameters. In the fitting process (see Sec. II) the selenium charge was kept fixed to the value of model $2(293 \mathrm{~K})$. An examination of Table IV indicates that the essential difference between this model and model $2(293 \mathrm{~K})$ is a certain decrease of the effective repulsive interactions K-O. This decrease is congruent with the volume contraction of the structure at $145 \mathrm{~K}$. The most conspicuous change in the phonon branches obtained for this model with respect to model $2(293 \mathrm{~K})$ can be seen in Fig. 3, where the lowest $\Sigma_{3}-\Sigma_{2}$ branch is shown. The experimental data at $145 \mathrm{~K}$ (Ref. 3) are also shown in the figure for comparison. The subtle change in the repulsive $\mathrm{K}-\mathrm{O}$ parameters is sufficient to produce a clear softening of the $\Sigma_{2}$ branch, which has now a strong minimum at $q=0.25 \mathrm{a}^{*}$. The corresponding mode eigenvector listed in Table $\mathrm{V}$ agrees even better than the one of model 2

TABLE VI. Elastic constants $\left(\times 10^{10} \mathrm{dyn} \mathrm{cm}^{2}\right)$ of $\mathrm{K}_{2} \mathrm{SeO}_{4}$ : experimental (Ref. 32) and calculated values for model 2 (293 K).

\begin{tabular}{llllllllll}
\hline \hline & $C_{11}$ & $C_{22}$ & $C_{33}$ & $C_{44}$ & $C_{55}$ & $C_{66}$ & $C_{12}$ & $C_{23}$ & $C_{13}$ \\
\hline Expt. & 50.5 & 47.9 & 40.7 & 8.1 & 14.2 & 15.4 & 15 & 19 & 17 \\
Calc. & 58.7 & 53.0 & 49.2 & 3.6 & 16.3 & 18.1 & 17.1 & 16.9 & 17.1 \\
\hline \hline
\end{tabular}


TABLE VII. Raman frequencies $\left(\mathrm{cm}^{-1}\right)$ of $\mathrm{K}_{2} \mathrm{SeO}_{4}$ : experimental (Ref. 6) and calculated values for model 2 (293 K).

\begin{tabular}{|c|c|c|c|c|c|}
\hline \multicolumn{3}{|c|}{$A_{g}$} & \multicolumn{3}{|c|}{$B_{1 g}$} \\
\hline$\omega_{\text {exp }}$ & $\omega_{\text {calc }}$ & Discrepancy $(\%)$ & $\omega_{\text {expt }}$ & $\omega_{\text {calc }}$ & Discrepancy $(\%)$ \\
\hline 41 & 43 & 4.7 & 47 & 81 & 72.3 \\
\hline 75 & 88 & 17.3 & 74 & 88 & 18.9 \\
\hline 97 & 94 & 3.1 & 96 & 102 & 6.2 \\
\hline 105 & 106 & 1.0 & 109 & 128 & 17.4 \\
\hline 121 & 125 & 3.3 & 120 & 146 & 21.7 \\
\hline 144 & 130 & 9.7 & 143 & 152 & 6.3 \\
\hline 162 & 162 & 0 & 160 & 162 & 1.2 \\
\hline \multicolumn{3}{|c|}{$B_{2 g}$} & \multicolumn{3}{|c|}{$B_{3 g}$} \\
\hline 50 & 57 & 14.0 & 44 & 40 & 10.0 \\
\hline 75 & 81 & 8.0 & 75 & 71 & 5.3 \\
\hline 95 & 85 & 10.5 & 93 & 82 & 11.8 \\
\hline 119 & 114 & 4.2 & 119 & 113 & 5.0 \\
\hline 146 & 154 & 5.5 & 142 & 122 & 14.1 \\
\hline
\end{tabular}

$(293 \mathrm{~K})$ with the experimental one.

The softening of the $\Sigma_{2}$ branch is specially sensitive to a variation of the repulsive parameter $A_{\mathrm{K}(\alpha)-\mathrm{O}(1-2)}$. The phonon branch obtained after a decrease of less than $4 \%$ $\left(102.6 \times 10^{3} \mathrm{Kcal} / \mathrm{mol}\right)$ of this parameter with respect to its value in model $2(145 \mathrm{~K})$ is also shown in Fig. 3. What is remarkable is the resulting deep minimum of the branch at a point $\left(q=0.27 \mathrm{a}^{*}\right)$, which is now closer to the experimental value of the soft-mode wave vector.

These results contrast with those reported in Ref. 12, where the formation of the deep minimum at values close to $\frac{1}{3} \mathbf{a}^{*}$ was attributed to a specific $\mathrm{O}-\mathrm{O}$ interaction. We performed additional calculations changing the $\mathrm{O}-\mathrm{O}$ repulsive force. The results demonstrated that in our

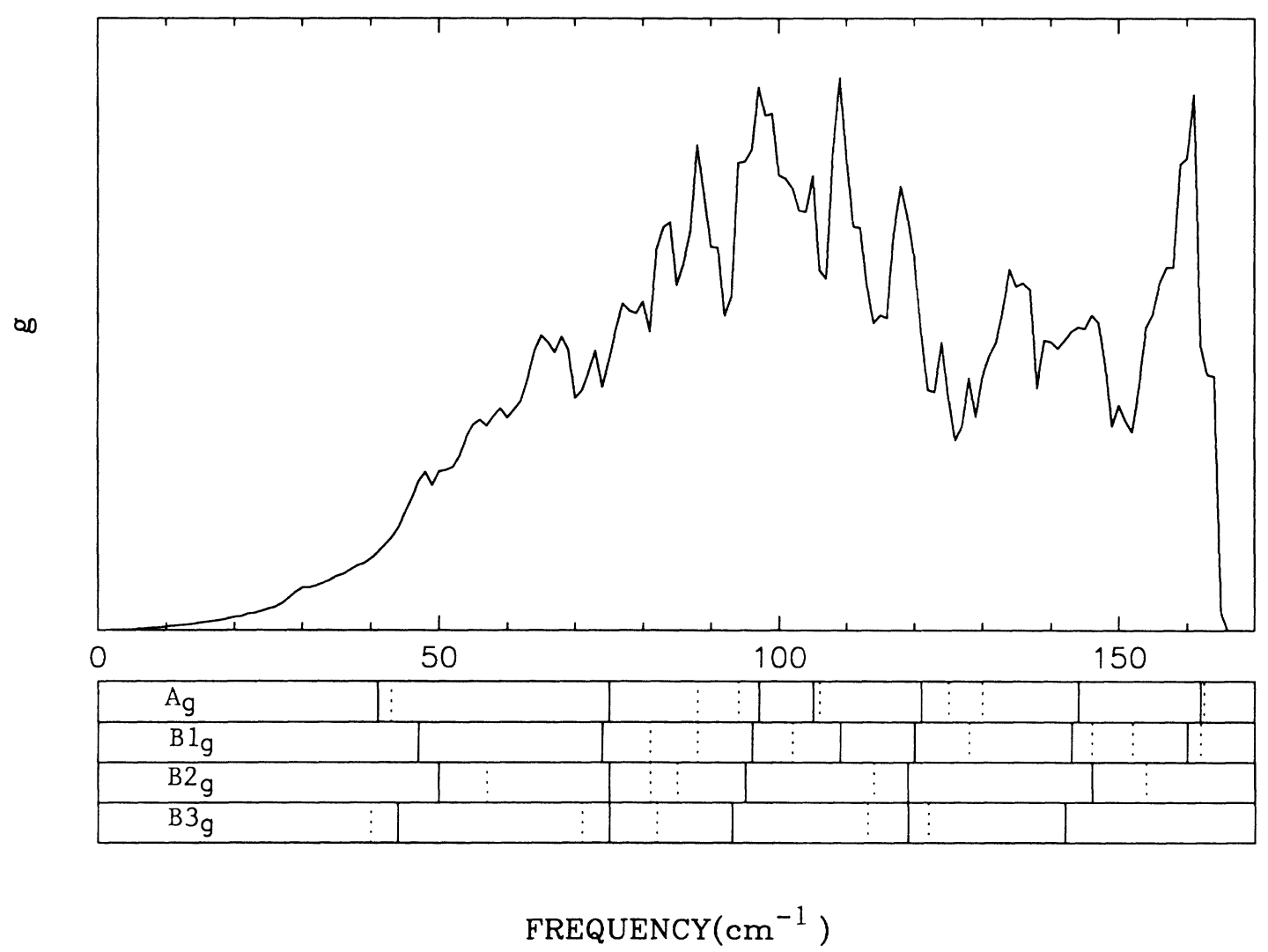

FIG. 4. Density of states of $\mathrm{K}_{2} \mathrm{SeO}_{4}$ corresponding to the external modes calculated with model 2 (293 K). Also a graphical comparison of the calculated Raman frequencies (dashed lines) and the experimental ones (Ref. 6) is shown. 

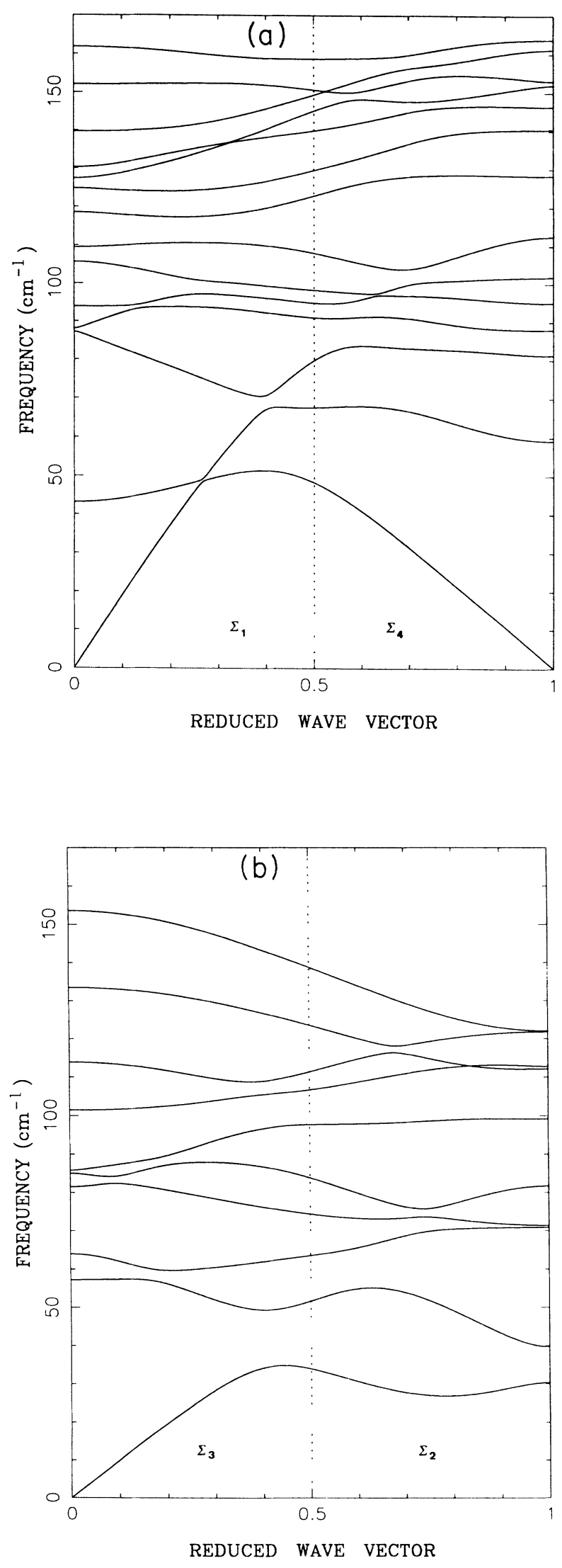

FIG. 5. Calculated phonon dispersion curves of $\mathrm{K}_{2} \mathrm{SeO}_{4}$ within model $2\left(293 \mathrm{~K}\right.$ ) for (a) the $\Sigma_{1}$ and $\Sigma_{4}$ and (b) the $\Sigma_{2}$ and $\Sigma_{3}$ external vibrations plotted in the extended zone scheme.

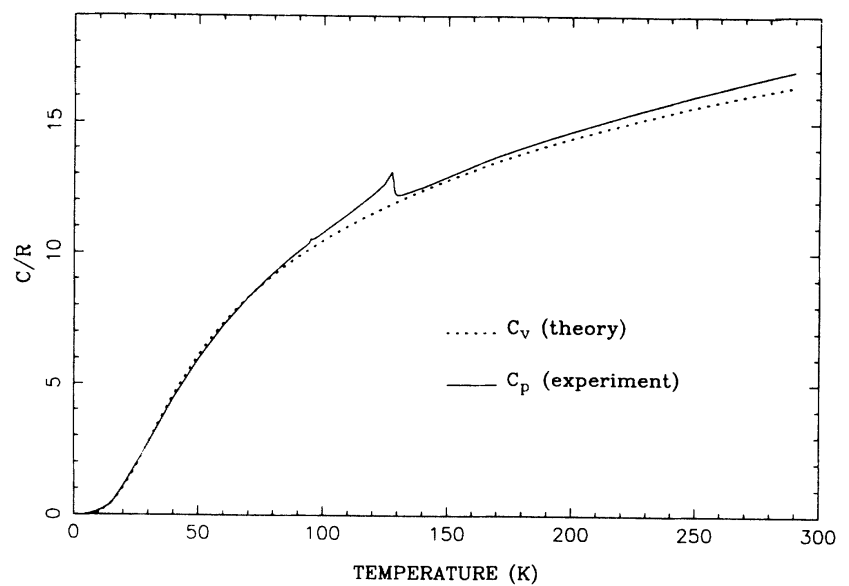

FIG. 6. Experimental constant-pressure specific heat (Ref. $34)$ and theoretical constant-volume specific heat (dotted line) of $\mathrm{K}_{2} \mathrm{SeO}_{4}$ calculated within model $2(293 \mathrm{~K})$.

model the sensitivity of the soft-phonon branch to changes of the $\mathrm{O}-\mathrm{O}$ repulsive interaction is rather weak.

In general, the following general effects could be observed: a decrease of either $\mathrm{O}-\mathrm{O}$ or $\mathrm{K}(\beta)-\mathrm{O}(1-2)$ repulsions tend to increase the frequencies of the soft branch, while the reverse is true for the $\mathrm{K}(\alpha)-\mathrm{O}(1-2)$ interaction, being also, as stressed above, a much stronger effect. On the other hand, we could observe that the relaxed structure depends rather weakly on precisely the latter interaction. This suggests that the static configuration of the structure is essentially caused by the contact between the other atoms, leaving the $\mathrm{K}(\alpha)$ atoms in cavities that are larger than their atomic size. Consequently, small changes in the repulsive parameters of these atoms (corresponding to small changes of effective atomic volume) do not cause significant variations of the equilibrium structure. On the other hand, the lattice modes with participation of these atoms can strongly depend on their repulsive interaction with the surrounding atoms. This interpretation is supported by the known experimental fact that the atom $K(\alpha)$ (or its equivalent in other $A_{2} B X_{4}$ materials) has systematically larger thermal displacement parameters than its homologous $\mathrm{K}(\beta)$.

\section{Pressure effects}

The pressure dependence of the soft $\Sigma_{2}$ branch was also investigated. With the model parameters fixed, the structure was relaxed using the WMIN program ${ }^{19}$ (see Sec. II) with a term $-p \Delta V$ added to the system energy. The phonon branches were then calculated for different pressures. Essentially, the effect of pressure is to raise the mode frequencies as a consequence of the negative volume change. Thus, the lattice modes have normal positive Gruneisen parameters, and the INC transition temperature decreases with pressure as observed experimentally. The strength of the pressure effect on the soft branch minimum strongly depends on its sharpness. For model $2(293 \mathrm{~K})$, the rate of change of the frequency minimum was $22 \mathrm{~K} / \mathrm{GPa}$ (Kelvin units are used for the frequency changes), while for model $2(145 \mathrm{~K})$ or its 
modification explained in Sec. III C 3 it could rise to values of the order of $50 \mathrm{~K} / \mathrm{GPa}$, which is of the same order of magnitude of the experimental pressure derivative of the INC transition temperature $(-59 \mathrm{~K} / \mathrm{GPa}){ }^{35}$

\section{5. $\mathrm{Cs}_{2} \mathrm{SeO}_{4}$}

From the above discussion, the effective size of the potassium atoms as compared with that of the selenate groups seems to play an essential role in the INC stability of $\mathrm{K}_{2} \mathrm{SeO}_{4}$. This assumption is further supported by the calculations we performed for $\mathrm{Cs}_{2} \mathrm{SeO}_{4}$.

In contrast with potassium selenate, the system is now stable for all lattice modes in model 1. In particular, the lowest $\Sigma_{2}$ branch has rather large real values and exhibits no minimum out of the center of the Brillouin zone (see Fig. 2). The corresponding zone-center mode has $A_{u}$ symmetry. The mode eigenvector at $0.31 \mathrm{a}^{*}$ keeps strong similarities with that calculated for the $\mathrm{K}_{2} \mathrm{SeO}_{4}$ models (see Table V). Especially the phases are practically identical. However, a very strong decrease of the rotation amplitudes of the tetrahedra can be observed, especially around the $a$ axis. Therefore, the larger size of the cesium atoms seems to cause a strong hinderance of the librations of the selenate groups, favoring the stability of the structure.

As in the case of potassium selenate, an increase of the inhomogeneity of the charge distribution in the selenate groups leads to a decrease of the frequencies of the $\Sigma_{2}$ branch and eventually to the appearance of a minimum close to $\frac{1}{3} \mathbf{a}^{*}$. However, the branch remains stable for all selenium charge values that have some reasonable degree of compatibility with the known equilibrium structure. The situation did not change qualitatively when a force model of the type of model $2(293 \mathrm{~K})$ was introduced.

\section{Selenate size}

As a further check of the role played by volume effects on the softening of the $\Sigma_{2}$ branch, the size of the selenate groups was artifically changed for $\mathrm{K}_{2} \mathrm{SeO}_{4}$. The interatomic distances $\mathrm{Se}-\mathrm{O}$ were augmented $20 \%$ and the structure was allowed to relax under model 2 . The subsequent vibrational analysis of the resulting structure showed that the system becomes unstable with several imaginary phonon branches along the line $\Sigma$, a $\Sigma_{2}$ branch with $B_{3 g}$ at the zone center being again the most unstable.

\section{CONCLUSIONS}

We have shown that the basic features of the lattice incommensurate instability of potassium selenate can be easily simulated by means of a simple semiempirical rigid-ion model, with the selenate groups reduced to rigid bodies. A force model consistent with the static structural data is enough for detecting in the subsequent phonon analysis of the system a single branch (of $\Sigma_{2}$ symmetry) unstable or tending to be unstable with a minimum close to $\frac{1}{3} \mathbf{a}^{*}$. The calculated eigenvector of the soft mode, which in principle would determine the structure of the INC modulation, is rather unsensitive to the details of the interatomic forces. The calculated eigenvectors have an excellent agreement with the experimental one, determined from the structural data of the INC phase. In particular, the phases of the complex amplitudes describing the eigenvector are virtually the same for all force models considered and coincide with the experimental ones. The similarities of the structural modulations of several $A_{2} B X_{4}$ compounds shown in Table I become then an obvious corollary of this property.

The semiempirical force model has only a few adjustable parameters (two or five depending on the case), which are fitted only using structural data. Such models are sufficient not only to mimic the INC unstable soft mode, but also to reproduce up to a certain degree the basic features of the vibrational properties.

The present analysis supports the idea that the origin of the INC instability observed in potassium selenate and other $A_{2} B X_{4}$ materials is essentially related with the specific characteristics of their static structure, somehow in the line of the "latent-symmetry" argument proposed in Ref. 9. However, the results obtained for the isomorphous $\mathrm{Cs}_{2} \mathrm{SeO}_{4}$, where no lattice instability is found, indicate that the mechanism cannot be reduced to a symmetry or pseudosymmetry argument, since the scale of the interatomic distances should also play a major role. Small changes in the repulsive forces can strongly vary the form of the soft-mode branch. It is specially significant how the branch minimum can be strongly sharpened by adjusting the force model to the structure observed at $145 \mathrm{~K}$, instead of the one at room temperature.

It can be observed that the formation of a minimum in the soft-mode branch is specially sensitive to the interaction $\mathrm{K}(\alpha)-\mathrm{O}(1-2)$. This contrasts with the previous study in Ref. 12, where the softening of the branch was simulated, changing an interaction O-O. Another important difference with this previous model is the charge distribution in the selenate groups. While in the present study no consistent force models could be found with selenium charges larger than $+1.6 e$, in Ref. 12 the charge of selenium was fixed to $+2 e$.

The effective size of the potassium $K(\alpha)$ plays an important role in the stabilization of the Pnam structure. Indeed, the substitution of potassium by the larger cesium atoms in $\mathrm{Cs}_{2} \mathrm{SeO}_{4}$ stabilizes the soft-mode branch and causes a significant change of its eigenvector, which reflects the stronger constraints on the rotations of the selenate tetrahedra. Also, the simulation made with artificially larger selenate groups points out the critical importance of the relative size of the different ionic groups with regard to the stability of the structure. The appearance in this case of several completely unstable $\Sigma$ branches agrees with the fact that the ionic tetrahedra are disordered in other compounds such as $\mathrm{Rb}_{2} \mathrm{ZnCl}_{4}$ or $\mathrm{K}_{2} \mathrm{ZnCl}_{4}$, where the ratio between the effective size of the tetrahedral groups and the $A$ cations is larger.

The procedure followed in the present work [a fit of the semiempirical force model through a SIMPLEX algorithm, ${ }^{18}$ using WMIN (Ref. 19) as a subroutine, followed by a calculation of the phonon branches by means of a program that allows us to include rigid bodies] can also 
be used to investigate other members of the family $A_{2} B X_{4}$. The present results suggest that by this means a prediction, at least qualitative, of the phase diagram of these compounds in terms of the effective size of the ionic units could be obtained.

Very recently a molecular dynamics study of potassium selenate using theoretical ("ab-initio") potentials has been reported in a preliminary form. ${ }^{36} \mathrm{~A}$ phase transition at a temperature close to the experimental ones $\left(T_{i}\right.$ or $\left.T_{c}\right)$ could be observed. The eventual INC character of the transition cannot be analyzed with such techniques. It would be interesting to compare the potentials, and in particular the charge distribution inside the selenate groups employed in that study, with those of the empirical models of the present work, but these data are missing in Ref. 36.

\section{ACKNOWLEDGMENTS}

This work was partially supported by the Spanish Dirección General de Investigación Científica y Técnica (DGICYT), Project No. PB87-0744. The authors wish to thank F. J. Zúñiga, T. Breczewski, and A. Arnaiz for providing prior to publication the structural data of $\mathrm{Cs}_{2} \mathrm{SeO}_{4}$.
${ }^{1}$ D. Axe, M. Iizumi, and G. Shirane, in Incommensurate Phases in Dielectrics: Part II, Materials, edited by R. Blinc and A. P. Levanyuk (North-Holland, Amsterdam, 1986), p. 1.

${ }^{2}$ H. Z. Cummins, Phys. Rep. 185, 211 (1990).

${ }^{3}$ M. Iizumi, J. D. Axe, G. Shirane, and K. Shimaoka, Phys. Rev. B 15, 4392 (1977).

${ }^{4}$ K. Itoh, A. Hinasada, H. Matsunaga, and E. Nakamura, J. Phys. Soc. Jpn. 52, 664 (1983).

${ }^{5}$ A. Kalman, J. S. Stephens, and D. W. J. Cruickshank, Acta Crystallogr. Sec. B 26, 1451 (1970).

${ }^{6}$ N. E. Massa, F. G. Ullman, and J. R. Hardy, Phys. Rev. B 27, 1523 (1983).

${ }^{7}$ V. Katkanant, J. R. Hardy, and F. G. Ullman, Ferroelectrics 82, 185 (1988).

${ }^{8}$ J. M. Pérez Mato, F. J. Zúñiga, and G. Madariaga, Phase Trans. 16/17, 439 (1989).

${ }^{9}$ V. Katkanant, P. J. Edwardson, J. R. Hardy, and L. Boyer, Phase Trans. 15, 103 (1989).

${ }^{10} \mathrm{~T}$. Janssen, Ferroelectrics 66, 203 (1986).

${ }^{11}$ A. Bussmann-Holder, H. Bilz, and G. Benedek, Phys. Rev. B 39, 9214 (1989).

${ }^{12}$ M. S. Haque and J. R. Hardy, Phys. Rev. B 21, 245 (1980).

${ }^{13}$ P. J. Edwardson, V. Katkanant, J. R. Hardy, and L. Boyer, Phys. Rev. B 35, 8470 (1987).

${ }^{14}$ J. M. Pérez-Mato, F. Gaztelua, G. Madariaga, and M. J. Tello, J. Phys. C 19, 1923 (1986).

${ }^{15}$ S. R. Cox, L. Y. Hsu, and D. E. Williams, Acta Crystallogr. Sec. A 36, 277 (1980).

${ }^{16}$ D. E. Williams and D. J. Houpt, Acta Crystallogr. Sec. B 42, 286 (1986); M. J. L. Sangster, U. Schroder, and R. M. Atwood, J. Phys. C 11, 1523 (1978).

${ }^{17}$ J. M. Pérez Mato, G. Madariaga, and F. J. Zúñiga, Phase
Trans. 16/17, 445 (1989).

18J. A. Nelder and R. Mead, Comput. J. 7, 308 (1965).

${ }^{19}$ W. R. Busing, Acta Crystallogr. Sec. A 28, S252 (1972).

${ }^{20}$ L. Y. Hsu and D. E. Williams, Acta Crystallogr. Sec. A 36, 277 (1980).

${ }^{21}$ S. L. Chaplot and K. R. Rao, Phys. Rev. B 35, 9771 (1987).

${ }^{22}$ CRC Handbook of Chemistry and Physics, edited by R. C. Weast (CRC, Cleveland, 1988), p. D-104.

${ }^{23}$ F. J. Zúñiga, T. Breczewski, and A. Arnaiz (unpublished).

${ }^{24}$ M. Wada, A. Sawada, Y. Ishibashi, and Y. Takagi, J. Phys. Soc. Jpn. 42, 1229 (1977).

${ }^{25}$ V. Fawcett, R. J. B. Hall, D. A. Long, and V. N. Sankaranarayanan, J. Raman Spectrosc. 2, 629 (1974).

${ }^{26}$ J. Petzelt, G. V. Kozlov, A. A. Volkov, and Y. Ishibashi, Z. Phys. B 33, 369 (1979).

${ }^{27}$ P. Echegut, F. Gervais, and N. E. Massa, Phys. Rev. B 30, 6039 (1984).

${ }^{28}$ P. Echegut, F. Gervais, and N. E. Massa, Phys. Rev. B 34, 278 (1986).

${ }^{29}$ G. Ventakataraman and V. C. Sahni, Rev. Mod. Phys. 42, 409 (1970).

${ }^{30}$ G. S. Pawley, Phys. Status Solidi B 39, 475 (1972).

${ }^{31}$ P. P. Ewald, Ann. Phys. (Leipzig) 64, 253 (1921).

${ }^{32}$ G. Hauret and J. P. Benoit, Ferroelectrics 40, 1 (1982).

${ }^{33}$ S. S. Ti, S. F. A. Kettle, and $\emptyset$. Ra, J. Raman Spectrosc. 5, 325 (1976).

${ }^{34}$ T. Atake, K. Nomoto, B. K. Chaudhuri, and H. Chinara, J. Chem. Thermodyn. 15, 383 (1983).

${ }^{35}$ J. P. Benoit, G. Hauret, Y. Luspin, A. M. Gillet, and J. Berger, Solid State Commun. 54, 1095 (1985).

${ }^{36}$ H. M. Lu and J. R. Hardy, Phys. Rev. Lett. 64, 661 (1990). 